\title{
Fundamentos sobre o Estudo da Dinâmica das Inovações no Agribusiness
}

\author{
Rosa Teresa Moreira Machado
}

\section{RESUMO}

O artigo é teórico-conceitual, no intuito de buscar uma explicação realista para a dinâmica das inovações em sistemas agroindustriais. Baseado em revisão bibliográfica, critica os fundamentos da teoria neoclássica que sustenta o modelo de inovação induzida, usado para interpretar o padrão de modernização agrícola conhecido por revolução verde. Assume a abordagem evolucionista como a mais adequada para explicar o caráter e o ritmo dessas inovações, a partir da definição de conceitos como paradigma tecnológico, trajetória tecnológica, ativos complementares, apropriabilidade das inovações e interdependência tecnológica setorial. Toma-se a indústria de carnes para ilustrar tal abordagem, identificando a direção mais incremental das inovações no agribusiness. As decisões estratégicas em P\&D das firmas de um sistema agroindustrial são limitadas por dependerem mais de avanços tecnológicos gerados noutros setores e por se inserirem numa estrutura de mercado que define a conduta das empresas. Mesmo assim, cada firma tem também sua trajetória específica de inovação, graças à aprendizagem própria e cumulativa de desenvolvimento e exploração de suas competências tecnológicas e organizacionais, e pode obter vantagens competitivas mediante estratégias como desenvolvimento de marca e de parcerias para suprimento e distribuição.

Palavras-chaves: abordagem evolucionista; agronegócio; inovação; estratégias das empresas.

\begin{abstract}
This article is theoretical and conceptual, aiming to a realistic explanation for the innovation dynamics in agricultural industrial systems. Based on bibliographical data, it critically reviews the foundations of the neoclassical theory that supports the induced innovation model, used for interpreting agricultural updating standard, known as the green revolution. It considers the evolutionist approach as being the most appropriate one to explain the character and the rhythm of these innovations, starting from the definition of concepts such as technological paradigm, technological trajectory, complementary assets, regimes of appropriability and technological interdependence among sectors. The beef industry is considered in order to illustrate such approach, identifying the incremental direction of innovations in agribusiness. Strategic decisions in R\&D of the companies of an agricultural industrial system are limited because they depend more heavily on the technological breakthroughs generated by other sectors, and because they are part of a market structure that defines the companies' behavior. Even then, each company has its specific innovation route, thanks to its own cumulative learning about development and the exploitation of its technological and organizational competencies, and can attain competitive advantages by means of strategies such as brand name development and partnerships for supply and distribution.
\end{abstract}

Key words: evolutionist approach; agribusiness; innovation; enterprises' strategies. 


\section{INTRODUÇÃO}

Inovação é um conceito bem mais amplo do que P\&D. Segundo Dosi (1988) a inovação trata de pesquisa, descoberta, experimentação, desenvolvimento, imitação e adoção de novos produtos, de novos processos de produção e novas formas organizacionais.

Neste artigo, busca-se compreender como se dá o processo de inovação no sistema agroindustrial, dentro de uma perspectiva sistêmica e realista das forças econômicas, institucionais e tecnológicas, que regem e condicionam as decisões dos agentes econômicos num ambiente de competição dinâmico. Seu conteúdo é de ordem conceitual e exploratório.

Iniciando com o referencial teórico tradicional, que ajudou a justificar a modernização do setor agropecuário e integrá-lo aos demais setores, o trabalho segue numa linha de abordagem denominada evolucionista para tentar explicar por que as inovações foram sendo delineadas dentro daquele padrão, baseado nos conceitos de paradigma tecnológico e trajetória tecnológica. Ao indicar que o ritmo e intensidade das inovações variam entre os setores produtivos, a análise é então direcionada para conceitualizar o sistema agroindustrial e entender por que os limites da inovação tecnológica são maiores nas firmas que compõem esse setor. Para ilustrar empiricamente a parte conceitual, faz uma breve identificação dos condicionantes e da direção do paradigma tecnológico da indústria de carnes, sem entrar em particularidades das estratégias competitivas de firmas dessa indústria.

O artigo finaliza com algumas considerações sobre a importância de cada firma traçar sua estratégia de inovação própria, aprendendo a explorar suas competências tecnológicas, incorporando inovações contínuas sob várias formas, como desenvolver uma organização apropriada para controlar ativos complementares e dominar a coordenação da cadeia produtiva, desenvolver a flexibilidade operacional, novas formas de suprimento e outras competências intangíveis, mais difíceis de serem copiadas.

\section{O Modelo de Inovação Induzida}

Para a teoria econômica neoclássica os preços são os grandes determinantes da competição. A inovação tecnológica é exógena às firmas e é tratada apenas como questão de alocação ótima de recursos escassos para satisfazer a demanda. Os 
tomadores de decisão são perfeitamente racionais e, portanto, maximizadores de resultados, já que atuam em ambiente de certeza, onde todos têm acesso às informações relevantes, numa estrutura concorrencial simétrica.

O modelo de inovação induzida de Hayami e Ruttan (1971), segundo Gomes (1986) e Silva (1984), trata de explicar a inovação tecnológica da agricultura dentro dessa perspectiva. As tecnologias biológico-químicas e as tecnologias mecânicas resultariam da necessidade de facilitar a substituição de fatores de produção relativamente escassos e, portanto, mais caros, por outros relativamente abundantes. Hayami e Ruttan (1971) apóiam-se em dados empíricos de uma determinada época histórica para sustentar esse modelo. No Japão, cuja escassez de terra era contrabalanceada com abundância de mão-de-obra, as inovações biológicoquímicas foram fundamentais para aumentar a produção agrícola. Nos Estados Unidos, as inovações mecânicas foram mais enfatizadas por causa da abundância de terras e escassez de mão-de-obra. Do mesmo modo, produtos com alta elasticidade de preço de demanda seriam mais priorizados por inovações. A pesquisa agrícola e o desenvolvimento tecnológico funcionariam, portanto, como resposta aos sinais de mercado, segundo o modelo de inovação induzida.

Esse modelo tem uma ótica produtivista, porque se dá sempre em função primordial de poupar fatores de produção agrícola. Nesse sentido, ele não é suficiente para explicar outros tipos de tecnologia que são desenvolvidas, por exemplo, para atender especificidades das relações intra e inter-setoriais (carne de porco com baixo teor de gordura), nem as ineficiências de se optar por tecnologias poupadoras de fatores que são abundantes em países menos desenvolvidos.

No modelo induzido, as instituições têm papel importante na criação de instrumentos para promover a disponibilidade de tecnologias modernas em prol do desenvolvimento rural, assim como se destaca o papel imprescindível do setor público na geração de tecnologias de fraca apropriabilidade, de pouco interesse para o setor privado ${ }^{(1)}$ (Gomes, 1986). Na década de 70, tal modelo, associado à interpretações desenvolvimentistas para o meio rural, serviu de referência teórica para justificar as ações públicas para expandir o padrão de modernização agrícola conhecido pelo nome de revolução verde.

Apesar do progresso técnico ser influenciado por forças econômicas, este modelo de inovações tem sido muito criticado sob vários aspectos a partir das limitações inerentes ao caráter estático, não histórico, inespecífico e de neutralidade dos pressupostos neoclássicos, assim como pela natureza contínua da função de produção, que admite perfeita substituição entre fatores, ou ainda pela forma mecanicista de tratar o processo de inovação. A estrutura de mercado e a tecnologia são dados exógenos, as firmas são passivas nessa estrutura e a inovação tecnológica é apenas resultado de uma escolha ótima de fatores dentro de um estoque de conhecimentos. 
As críticas à teoria neoclássica relacionadas às características do processo de inovação vêm de muitos estudiosos, dando origem a uma nova abordagem evolucionária, de inspiração schumpeteriana, cujos argumentos se vão complementando uns aos outros. Segundo Dosi (1988), um dos seus expoentes, conta com a contribuição de vários economistas entre os quais Rosenberg, Nelson e Winter, Freeman e Pavitt, para tentar uma interpretação sobre como se dá a inovação tecnológica em relação aos avanços científicos, de um lado, e ao processo de mercado, de outro. Outros autores como Penrose, Porter e Teece fazem parte desse conjunto de teóricos que questionam os pressupostos neoclássicos e analisam a importância da conduta da firma, seu papel estratégico e como se dão as inovações em ambientes competitivos dinâmicos. Ao revisar a literatura sobre esse tema, interessa buscar elementos teóricos para verificar se existem ambientes competitivos mais ou menos favoráveis a $\mathrm{P} \& \mathrm{D}$ para, em última instância, localizar esse processo no agribusiness.

\section{O Enfoque Evolucionista da Inovaçāo}

A abordagem evolucionista faz uma analogia com a teoria biológica de seleção natural para mostrar que as inovações não são decorrentes de um mecanismo racional de seleção de mercado, mas fortemente determinadas pela base tecnológica acumulada, num mundo onde, contrariamente aos pressupostos da teoria neoclássica, domina a incerteza, a racionalidade limitada, a diversidade de práticas estratégicas e comportamentais entre as firmas e ambigüidades entre os agentes econômicos.

As idéias básicas dos evolucionistas têm uma perspectiva temporal e até mesmo um determinismo histórico, à medida que as escolhas tecnológicas adquirem certa irreversibilidade em decorrência do caráter cumulativo e progressivo do desenvolvimento tecnológico. Ajudam também a compreender a importância das forças da demanda e da oferta (demand pull x technology push) no processo de inovação. Além disso, realçam a importância das condições institucionais que governam os interesses dos agentes econômicos (incluindo as formas de regulamentação, condições políticas, valores e comportamentos dominantes, estabelecimento de práticas de cooperação x competição) na definição dos padrões das mudanças (Dosi e Orsenigo, 1988).

Há também uma dimensão de interdependência micro x macro no processo de mudança tecnológica, já que o avanço tecnológico é endógeno às firmas, como também depende de externalidades inerentes às tecnologias de caráter público e coletivo, tão importantes para proporcionar complementaridades tecnológicas e 
sinergias que beneficiem setores e firmas em países e regiões, e tendem a ser internalizadas pelas firmas individualmente. Essas condições contextuais podem decorrer de ações não intencionais de organização ambiental (a exemplo do Silicon Valley) e/ou como fruto de estratégias explícitas de instituições públicas e privadas para desenvolver setores e regiões (Dosi, 1988).

Para os evolucionistas, as atividades de inovação não são aleatórias mas fortemente seletivas, seguindo um mecanismo de busca e seleção dentro da lógica de mercado, que possibilita inovações contínuas e cumulativas em função do estadoda-arte das tecnologias já em uso e da capacidade de cada firma para conjugar os vários tipos de conhecimento acumulados.

Parte desses conhecimentos acumulados são desenvolvidos em organizações formais como universidades e laboratórios de $\mathrm{P} \& \mathrm{D}$ públicos, explicitados em manuais e publicações técnico-científicas de difusão ampla; outros são conhecimentos privados e protegidos por patentes; há também aqueles conhecimentos técnicos tácitos, mais difíceis de serem transmitidos, porque são implícitos, intangíveis e apropriados pelas pessoas e/ou específicos às firmas, apreendidos informalmente pela prática do learning-by-doing e learning-by-using.

Interpretando dois conceitos complementares de Dosi (1988), pode-se afirmar que as inovações tecnológicas pontuais são frutos da evolução de uma trajetória tecnológica que, hierarquicamente, seriam alternativas incrementais subordinadas a um paradigma tecnológico. Ou seja, enquanto o paradigma tecnológico se refere a "um padrão de solução de problemas técno-econômicos selecionados, baseado em princípios altamente selecionados derivados das ciências naturais" (Dosi, 1988, p. 225) capaz de definir tipos de artefatos básicos que podem ser desenvolvidos e aperfeiçoados, a trajetória tecnológica define as oportunidades tecnológicas para inovações posteriores, a partir do conceito tecnológico central dado pelo paradigma, tal como o motor de combustão interna na indústria automobilística e o chip da indústria microeletrônica.

Dosi (1988) chama a atenção para a diferença entre mudanças tecnológicas dentro de um dado paradigma e mudanças de paradigma. No primeiro caso, os padrões de inovação seguem as trajetórias normais definidas pelos limites de um dado paradigma e são condicionados por fatores ambientais, como a demanda e os preços relativos. Já as mudanças de paradigma dependem fundamentalmente de avanços da ciência e das tecnologias gerais públicas, e representam descontinuidades maiores nos padrões de mudança. Quando acontecem avanços tecnológicos superiores a um velho paradigma, irreversivelmente muda-se o paradigma, independentemente do nível dos preços dos inputs. Há, portanto, diferentes níveis de padrões de inovação. 
Esse enfoque é de natureza schumpeteriana, ao evidenciar que o motor da dinâmica capitalista está na capacidade de se gerar e difundir inovações - em produto, processo, organizacional e/ou mercadológica, num jogo competitivo em que as empresas buscam conseguir assimetrias que lhes garantam vantagens competitivas em face dos concorrentes. A exploração das competências de cada firma, a sua história de aprendizagem e as estratégias de cada uma delas são importantes para explicar o comportamento microeconômico, bem como as transformações do ambiente competitivo. Cada firma tem também sua trajetória específica de inovação, que depende de aprendizagem local, específica e cumulativa de desenvolvimento e exploração de suas competências tecnológicas.

\section{As Diferenças Setoriais em Inovação}

Para os evolucionistas, a capacidade de inovação, além de ser assimétrica entre as firmas, varia entre países, entre setores específicos e mesmo no tempo.

Dosi (1988) identifica algumas razões para explicar essas variações dos avanços tecnológicos. As oportunidades tecnológicas específicas de um paradigma são um primeiro determinante das diferenças nas taxas de inovação. Esforços para inovar são também uma função da estrutura da demanda e das condições de apropriabilidade dos lucros gerados por uma inovação. Os agentes privados só irão investir numa oportunidade tecnológica, se houver mercado disposto a pagar por isso. Por outro lado, as condições de apropriabilidade diferem entre indústrias e entre tecnologias.

Teece (1986) também discute a capacidade de uma empresa obter retornos econômicos com inovações pioneiras, a partir dos fatores ambientais que definem o regime de apropriação. Para ele, o regime de apropriabilidade de uma inovação é mais forte, quando ela é mais difícil de ser copiada pelos competidores. Isso vai depender do grau de conhecimento tácito embutido na natureza da tecnologia e da eficácia dos mecanismos de proteção legal aos direitos de propriedade sobre inovações, a exemplo das patentes, dos direitos autorais, dos segredos comerciais e das marcas registradas. Pela leitura do artigo, fica claro que os trade secrets das fórmulas e processos têm tido apropriabilidade mais forte que as patentes.

Ainda dentro dessa questão, Teece (1986) apresenta mais dois conceitos interrelacionados: o paradigma do projeto dominante e os ativos complementares. À luz dos trabalhos de Kuln, Dosi e Utterback, o paradigma do projeto dominante refere-se ao último estágio de evolução de uma inovação, quando um determinado produto conquista a preferência do mercado e passa a ser o modelo de referência para os competidores. Definido o projeto dominante, a competição entre as empresas migra do design para o preço, de tal forma que as economias de escala e aprendizagem, visando a reduzir custos unitários, passam a ser mais 
importantes. Nessa etapa, os imitadores podem construir posições mais vantajosas que o inovador, ao se capacitarem em competências e ativos complementares: imagem da marca, domínio dos canais de distribuição, suporte pós-venda.

Para Teece (1986), os ativos complementares podem ser genéricos, quando não são inflexíveis a ponto de serem unicamente usados na inovação em exame; especializados, quando são desenvolvidos sob medida para a empresa inovadora e com isso gerando uma relação unilateral de dependência, ou co-especializados, onde a relação de dependência é bilateral.

Preocupado com o problema de garantir um regime de apropriabilidade favorável ao inovador a partir da etapa em que os ativos complementares passam a ser mais relevantes, Teece (1986) incorpora uma análise sobre formas contratuais mais adequadas para controlar inovações que envolvem esses ativos ${ }^{(2)}$. Na presença de ativos especializados e co-especializados, o inovador pode internalizar o desenvolvimento e a produção dos ativos complementares por meio da integração vertical, passando a fazer tudo sozinho (manufatura, distribuição, serviços e tecnologias complementares); ou, num outro extremo, obter esses ativos via relações contratuais. Enquanto a completa integração seja proibitivamente cara e não garanta que a empresa possa ser boa em tudo, relacionamentos contratuais estão sujeitos à sorte, porque apenas uma, ou ambas as partes terão de comprometer capital em investimentos irreversíveis, que perderão valor, caso a relação se desfaça. Na maioria das vezes predomina um modelo misto, uma forma contratual intermediária entre as soluções extremas de integrar ou licenciar.

O dilema entre integrar ou terceirizar $\mathrm{P} \& \mathrm{D}$ em inovações que envolvem ativos complementares é uma decisão estratégica relacionada à capacidade de apropriação de lucros com essas inovações. Se os métodos legais de proteção das inovações não são tão eficientes para impedir imitadores, há maior necessidade de integrar ativos co-especializados, pois quem tiver controle desses ativos terá vantagens sobre os concorrentes. A parceria em $\mathrm{P} \& \mathrm{D}^{(3)}$ é uma forma ideal quando a tecnologia do inovador está bem protegida e existem diferentes parceiros alternativos, oferecendo o mesmo suprimento para o inovador (Teece, 1986).

Teece (1986) cita o caso do adoçante aspartame, introduzido no mercado pela Searle's em duas versões: Nutrasweet para clientes industriais e Equal para consumidor final. A Searle's conseguiu estabelecer um regime de forte apropriação dessa inovação estabelecendo: 1) um contrato de parceria com a Ajinomoto, para desenvolver o produto e ter acesso aos conhecimentos mais tácitos em biotecnologia; 2) construção de ativos complementares mediante uma estratégia de desenvolvimento de marca, associada a uma patente de 17 anos, com uma prorrogação adicional de 5 anos; 3 ) integração da manufatura do produto para evitar concorrência de possíveis competidores. Expirada a patente, a despeito dos competido- 
res, a empresa inovadora continuou a dominar o mercado de aspartame por ter conseguido fixar a reputação da sua marca entre os clientes.

Para Teece (1986) não existe estratégia ótima que garanta o sucesso de uma empresa inovadora. É claro, porém, que as decisões de investir em P\&D não podem estar desligadas da análise estratégica de mercados e da posição da firma na indústria. As maiores empresas têm mais chance de já terem ativos especializados e co-especializados ao lançar novos produtos, em relação às pequenas empresas, bem como de alavancar parcerias e coalizões bem sucedidas. A propriedade de ativos especializados é fundamental para entender quem ganha e quem perde com a inovação.

Num estudo empírico para classificar setores segundo as origens da tecnologia, as necessidades dos usuários dos produtos e as formas de apropriação das inovações, Pavitt (apud Dosi, 1988), identificou 4 grandes grupos de setores: os setores dominados por fornecedores, os de escala intensiva, os de fornecedores especializados e os baseados em ciência.

Pela caracterização de Pavitt (apud Dosi, 1988), pode-se afirmar que as atividades agropecuárias e agroindustriais estariam mais identificadas com a categoria dos setores dominados por fornecedores, embora Dosi (1988), ainda se referindo a Pavitt, inclua a indústria de produtos alimentícios nos setores de escala intensiva. A origem das tecnologias dos setores dominados por fornecedores é os fornecedores de equipamentos e insumos. As firmas do próprio setor fazem pouco investimento em P\&D. Dominam as inovações de processo (pela absorção de inovações geradas em outros setores), e sua absorção decorre da busca de reduzir custos pelas empresas e aumentar a eficiência dos fatores. A apropriabilidade é baixa. Nos setores de escala intensiva as firmas investem fortemente em P\&D e produzem internamente boa parte do seu processo tecnológico. As inovações são em produtos e processos. As atividades produtivas são mais complexas, com economias de escala de vários tipos (em produção, design, P\&D). A apropriabilidade é maior, tanto quanto o tamanho das firmas que tendem à integração vertical. O processo produtivo desse grupo de setor pode ser em linha de montagem (automóveis, eletrodomésticos) ou de processamento contínuo (cimento, produtos alimentícios).

\section{Os Enfoques Conceituais do Sistema Agroindustrial e os Limites em P\&D desse Setor}

Pela tipologia setorial de inovações de Pavitt (apud Dosi, 1988), pode-se entender que o comportamento competitivo das firmas tem fortes ligações com o fenômeno macroeconômico das interdependências industriais e tecnológicas entre os 
setores. O padrão de produção e uso da inovação variaria tanto quanto as características setoriais. Os sistemas agroindustriais seriam, portanto, mais absorvedores de tecnologias geradas noutros setores do que geradores de tecnologia própria.

A noção de interdependência tecnológica intersetorial remonta ao conceito da matriz insumo-produto, enfoque posteriormente desenvolvido dentro de uma perspectiva sistêmica até se chegar a duas diferentes visões do agribusiness: o enfoque da escola de Harvard, de Davis e Goldberg (apud Zylbersztajn, 1995) e o de cadeia agro-alimentar ou filière (Zylbersztajn, 1995) .

Davis e Goldberg, (apud Zylbersztajn, 1995, p. 106) definem o termo agribusiness como "a soma de todas as operações associadas à produção e distribuição de insumos agrícolas, operações realizadas nas unidades agrícolas bem como as ações de estocagem, processamento e distribuição dos produtos, e também dos produtos derivados". Segundo Dosi e Orsenigo (1988), a tradição francesa tenta capturar o aspecto da estrutura relativamente ordenada da hierarquia tecnológica a que estão sujeitos os agentes pertencentes a um mercado, com o conceito de filière, que é um grupo de setores que são conectados por fortes interligações comportamentais e tecnológicas. Para Morvan (apud Zylbersztajn, 1995 p. 125), "cadeia ("filière") é uma seqüência de operações que conduzem à produção de bens. Sua articulação é amplamente influenciada pela fronteira de possibilidades ditadas pela tecnologia e é definida pelas estratégias dos agentes que buscam a maximização dos seus lucros. As relações entre os agentes são de interdependência ou complementariedade e são determinadas por forças hierárquicas. Em diferentes níveis de análise a cadeia é um sistema, mais ou menos capaz de assegurar sua própria transformação".

Segundo Zylbersztajn (1995), esses dois enfoques partem da versão mais tradicional da Teoria de Organização Industrial, conhecida como o paradigma Estrutura-Conduta-Desempenho. Para identificar mudanças mais significativas nos sistemas agro-industriais, alguns aspectos dinâmicos como as inovações tecnológicas podem ser introduzidos à medida que são importantes para modificar produtos e, "em consequência, a própria estrutura dos mercados" (Zylbersztajn, 1995 p. 126). A variável tecnologia recebe tratamento especial mas diferenciado em ambos os enfoques. Enquanto a literatura de cadeias tem perspectiva schumpeteriana, o estudo das mudanças tecnológicas no modelo de Harvard é limitado, porque é de concepção neoclássica, restringindo-se às inovações induzidas por mudanças nos preços dos fatores.

As decisões estratégicas em inovação das firmas de um sistema agroindustrial têm, portanto, seus limites, à medida que pertencem a um setor de menor dinamismo tecnológico e por se inserirem em contexto, cuja estrutura de mercado define a conduta das empresas. 


\section{O Paradigma Tecnológico da Indústria de Carnes}

O referencial teórico dos autores evolucionistas enfatiza que a dinâmica tecnológica é específica para cada setor industrial. Procurando entender como se dá esse processo no sistema agroindustrial, este item apresenta as principais características das inovações tecnológicas da indústria de carnes, baseado no trabalho de Campos (1996).

No nível do desenvolvimento conceitual até aqui registrado, as estratégias competitivas das firmas concorrentes numa mesma cadeia produtiva relacionadas a inovações estariam definidas dentro dos limites das oportunidades tecnológicas do paradigma tecnológico dominante do respectivo setor.

$\mathrm{Na}$ indústria de carnes as inovações tecnológicas do produto para satisfazer as necessidades dos consumidores têm sido de natureza incremental, direcionadas à solução de trade-offs entre sabor, textura, cor, rapidez e conveniência no consumo, qualidades nutricionais, possibilidades de conservação e facilidades na distribuição.

Para tanto, à acumulação paulatina de conhecimentos também incrementais no nível do que já existia em termos de processo de produção, foram sendo incorporados: (1) adaptação de técnicas já conhecidas de conservação (cura, defumação, congelamento) para o processamento de alimentos em grande escala, mediante o desenvolvimento de equipamentos específicos e a ampliação do campo de conhecimento científico no apoio às tecnologias de conservação; (2) desenvolvimento de novas técnicas de conservação (irradiação e desidratação a frio), no âmbito mais geral da indústria de alimentos, com algumas inovações radicais originárias de programas de $\mathrm{P} \& \mathrm{D}$.

As tecnologias de conservação e processamento de carnes exigem uma base de conhecimentos específicos dentro do campo científico da química, biologia e microbiologia, de modo que o desenvolvimento tecnológico dessa indústria depende de uma organização institucional pública e privada também específica em pesquisa básica e aplicada.

Confirmando a classificação de Pavitt (apud Dosi, 1988), essa indústria incorpora importantes inovações geradas incessantemente fora do setor, muitas delas de ponta, como a engenharia genética e as técnicas de biologia molecular e as tecnologias microeletrônicas, com efeitos importantes para os rendimentos do processo e qualidade dos produtos. "As tecnologias de processamento transformamse continuamente pela absorção de novos insumos derivados das novas biotecnologias e de equipamentos automatizados ou de novas técnicas 
organizacionais, caracterizando importantes inovações em processos decorrentes de inovações em outras indústrias" (Campos, 1996, p. 284).

A dinâmica tecnológica não se restringe, porém, às inovações provenientes de outros setores; à medida que as inovações e aprimoramento das técnicas de processo se ampliam, surgem novas oportunidades para desenvolver outros produtos com melhores qualidades organolépticas, nutricionais e de maior conveniência para consumo, ampliando as oportunidades mercadológicas com produtos de maior valor agregado e possibilidades de maior aproveitamento da matéria-prima básica. Aquelas empresas de maior fôlego passam então a investir em tecnologia própria, criando condições internas para ampliar as oportunidades tecnológicas oferecidas pelo paradigma. "O desenvolvimento das técnicas de manuseio das carnes no processo de abate, a "desossa a quente" e o desenvolvimento de infra-estrutura e capacitação para $P \& D$ nas empresas indicam o esforço de capacitação através de formas internas de aprendizagem, para o desenvolvimento de produtos e processos" (Campos, 1996, p. 285). As empresas líderes da indústria de carnes, trabalhando com economias de escala e investindo em $P \& D$, segundo os critérios de classificação de Pavitt (apud Dosi, 1988) estariam, portanto, dentro do setor de escala intensiva.

Campos (1996) constata, no entanto, que os avanços mais recentes incorporados na indústria de carnes, relacionados às áreas científicas de ponta, não são tão profundos, a ponto de alterarem as bases tecnológicas dos processos produtivos. Com isso, não havendo descontinuidades maiores das trajetórias tecnológicas, as firmas podem ser afetadas pela inércia das rotinas do paradigma ou serem mais estimuladas a aprimorar suas atividades de aprendizagem tecnológica. Contudo seu trabalho não entra nas particularidades das estratégias competitivas de firmas ${ }^{(4)}$.

\section{Algumas Considerações Finals sobre Estratégia de Empresas}

Tentando arrematar as idéias, as decisões estratégicas em termos de P\&D das firmas de um sistema agroindustrial são limitadas, na maioria das vezes de natureza incremental. Além de dependerem mais de avanços tecnológicos gerados noutros setores, inserem-se em contexto, cuja estrutura de mercado define a conduta das empresas. Mesmo assim, repetindo as idéias de Dosi (1988), cada firma tem também sua trajetória específica de inovação, que depende de aprendizagem local, específica e cumulativa de desenvolvimento e exploração de suas competências tecnológicas. 
A competitividade entre firmas concorrentes de uma mesma indústria passa pela questão da inovação tecnológica mas não se limita a isso. Como explicar, por exemplo, por que duas firmas, localizadas num mesmo setor produtivo, têm comportamentos distintos acerca do processo de inovação? Umas internalizam P\&D; outras fazem alianças estratégicas. As ações inovadoras vão além da incorporação de tecnologias propriamente ditas. Estão também associadas às formas de organização das firmas, às competências adquiridas em ativos complementares, como a imagem e o domínio da coordenação da extensão da cadeia produtiva.

As inovações não se restringem a investimentos em equipamentos avançados mas dependem da capacidade da empresa explorar o potencial criativo dos recursos produtivos tangíveis e disponíveis combinados com outros menos tangíveis, como habilidades, experiência acumulada e conhecimentos incorporados pelo seu capital humano. A nova onda do just-in-time, zero-defeitos e controle de qualidade total não é, por exemplo, suficiente para a empresa reduzir custos e melhorar a qualidade e flexibilidade. Como são programas que também podem ser copiados por competidores, a empresa só tem sucesso competitivo no longo prazo, quando consegue ter capabilidades de combinar recursos em nível de chão-de-fábrica. A confiança e a flexibilidade estratégica se constrói no processo de operações, por meio de aprendizagem organizacional e experimentação como, por exemplo, desenvolver rapidamente produtos a baixo custo com o mínimo de recursos e/ou mudar rapidamente a linha de produtos em função da demanda. É no processo e no resultado do sistema de operações que se conseguem vantagens competitivas (Wheelwright e Hayes, 1995).

Para uma empresa de uma dada indústria, o conceito tradicional de estratégia competitiva está relacionado com as decisões que ela toma para se ajustar às forças competitivas e encontrar uma posição, em que tais forças lhe possam ser favoráveis (Porter, 1989). Essas estratégias são variadas tanto como os tipos de negócio e os objetivos perseguidos. Num ambiente turbulento, onde as forças competitivas assumem dimensão globalizante, a empresa é competitiva se conseguir assimetrias em forma de vantagem competitiva, por algum tipo de inovação, em produto, em processo, em novas fontes de suprimento, em novas oportunidades de mercado e formas organizacionais alternativas mais eficientes, sem prejuízo da qualidade e do preço. No entanto a vantagem competitiva só é mantida mediante melhorias contínuas, visto que a maioria das inovações tendem a ser copiadas pelos rivais.

A idéia mais recente de estratégia competitiva implica, portanto, escolher-se a forma de competição e desenvolver uma organização apropriada. A estratégia de operações passa a ser entendida como suporte das competências essenciais da empresa e realimenta a construção de novas aptidões que a distinguem dos con- 
correntes. A manufatura passa a ser processo integrado do negócio, onde se cria o valor do negócio (Drucher, 1995). A firma passa a ser agente estratégico ativo que procura superar com as restrições através das inovações (Best, 1990).

\section{NOTAS}

${ }^{1}$ A pesquisa básica e tecnologias de processos e produtos de domínio público tais como sementes melhoradas de variedade pura, criação de novos métodos de preparo de solo e plantio, espaçamento e manejo de animais devem ficar a cargo da pesquisa pública. As organizações privadas se interessam por tecnologias em produtos, protegidas por patentes e comercializáveis, como sementes híbridas, animais desenvolvidos geneticamente, adubos, defensivos, produtos químico-veterinários, máquinas e equipamentos diversos.

${ }^{2}$ Embora não mencione a economia dos custos de transação que trata das estruturas de governança na presença de especificidade de ativos, as idéias de Teece (1986) se assemelham às de Williamson (1985).

${ }^{3}$ Muitos autores discutem a importância de alianças estratégicas para conseguir vantagens competitivas e suas dificuldades e limitações (Porter, 1990; McFarlan e Nolan, 1995; Bleeke e Ernst, 1995).

${ }^{4}$ Os trabalhos de Matos (1996), Mior (1992) e de Jank (1996) tratam dessa temática sob abordagens teóricas distintas.

\section{REFERENCIAS BibLIOGRÁFICAS}

BEST, M. H.

The new competition : institutions of industrial restructuring. Cambridge : Harvard University Press, 1990.

BLEEKE, J.;

ERNST, D.

Is your strategic alliance really a sale? Harvard Business Review, p. 97-105, Jan./Feb. 1995.

CAMPOS, R. R.

$\mathrm{O}$ paradigma tecnológico da indústriạ de carnes. Iñ: XIX SIMPÓSIO DE GESTÃO DA INOVAČ̃̃ TECNOLÓGICA (1996 : São Paulo). Anais ... São
Paulo : USP/PGT/FIA/PACTo, 1996. p. 282-299.

DOSI, G.

The nature of the innovative process. In: DOSI, G. et al (Eds.). Technical change and economic theory. London: Pinter, 1988. p. 221-238.

DOSI, G., ORSENIGO, L.

Coordination and transformation : an overview of structures, behaviours and change in evolutionary environments. In: DOSI, G. et al (Eds.). Technical change and economic theory. London : Pinter, 1988. p. 13-37. 
DRUCHER, P. F.

The emerging theory of manufacturing. In: PISANO, G. P.; HAYES, R. H. (Eds.). Manufacturing renaissance. Cambridge : Harvard Business School Publishing, 1995. v. 1. p. 47-60.

GOMES, S. T.

Condicionantes da modernização do pequeno agricultor. São Paulo : IPE-USP, 1986.

HAYAMI, Y.;

RUTTAN, V. W.

Agricultural development : and international perspective. Baltimore : John Hopkins Press, 1971.

JANK, M. S.

Competitividade do agribusiness brasileiro : discussão teórica e evidências no sistema carnes. São Paulo, 1996. Tese (Doutorado) - Faculdade de Economia, Administração e Contabilidade, Universidade de São Paulo.

\section{MATOS, V.}

Estratégias competitivas na avicultura brasileira : o caso das empresas líderes. In: WORKSHOP TEORICO SOBRE ECONOMIA POLITICA DA AGRICULTURA (1996 : Campinas). Anais ... Campinas : 1996. p. 476506.

MCFARLAN, F. W.;

NOLAN, R. L.

How to manage an IT outsourcing alliance. Sloan Management Review, p. 09-23, Winter 1995.
MIOR, L. C.

Empresas agroalimentares, produção agrícola familiar e competitividade no complexo carnes de Santa Catarina. Rio de Janeiro, 1992. Dissertação (Mestrado) - Instituto de Ciências Humanas e Sociais, Universidade Federal Rural do Rio de Janeiro.

PORTER, M. E.

The competitive advantage of nations. Harvard Business Review, p. 73-93, Mar./Apr. 1990.

Vantagem competitiva. Rio de Janeiro : Campus, 1989.

SILVA, G. L. S. P. DA.

Produtividade agrícola, pesquisa e extensão rural : evolução e determinantes da produtividade agrícola. São Paulo : IPE-USP, 1984.

TEECE, D. J.

Profiting from technological innovation : implications for integration, collaboration, licensing and public policy. Research Policy, v. 15, p. 285305, 1986.

WHEELWRIGHT, S. C.; HAYES, R. H.

Competing through manufacturing. In: PISANO, G. P.; HAYES, R. H. (Eds.). Manufacturing renaissance. Cambridge : Harvard Business School Publishing, 1995. v. 1. p. 03-24. 
WILLIAMSON, O. E.

The economic institutions of capitalism. New York : Free Press, 1985.

ZYLBERSZTAJN, D.

Estruturas de governança e coordenação do agribusiness : uma aplicação da nova economia das instituições. São Paulo, 1995. Tese (Livre Docência) - Faculdade de Economia, Administração e Contabilidade, Universidade de São Paulo. 